Proyecciones

Vol. 24, $\mathrm{N}^{o}$ 1, pp. 49-63, May 2005.

Universidad Católica del Norte

Antofagasta - Chile

\title{
SPECTRAL PROPERTIES OF A NON SELFADJOINT SYSTEM OF DIFFERENTIAL EQUATIONS WITH A SPECTRAL PARAMETER IN THE BOUNDARY CONDITION
}

E. KIR a., G. BASCANBAZ-TUNCA b. C. YANIK c.

a. Gazi University, Turkey

b. Ankara University, Turkey

c. Hacettepe University, Turkey

Received : October 2004, Accepted : April 2005.

\begin{abstract}
In this paper we investigated the spectrum of the operator $L(\lambda)$ generated in Hilbert Space of vector-valued functions $L^{2}\left(\mathbf{R}_{+}, \mathbf{C}_{2}\right)$ by the system

$(0.1) \mathrm{iy}_{1}^{\prime}+\mathrm{q}_{1}(x) \mathrm{y}_{2}=\lambda \mathrm{y}_{1}, \quad-\mathrm{iy}_{2}^{\prime}+\mathrm{q}_{2}(x) \mathrm{y}_{1}=\lambda \mathrm{y}_{2}, \quad \mathrm{x} \in \mathbf{R}_{+}:=[0, \infty)$, and the spectral parameter- dependent boundary condition

$$
\left(a_{1} \lambda+b_{1}\right) y_{2}(0, \lambda)-\left(a_{2} \lambda+b_{2}\right) y_{1}(0, \lambda)=0,
$$

where $\lambda$ is a complex parameter, $q_{i}, i=1,2$ are complex-valued functions

$a_{i} \neq 0, b_{i} \neq 0, i=1,2$ are complex constants. Under the condition $\sup _{x \in R_{+}}\left\{\exp \varepsilon x\left|q_{i}(x)\right|\right\}<\infty, i=1,2, \varepsilon>0$,

we proved that $L(\lambda)$ has a finite number of eigenvalues and spectral singularities with finite multiplicities. Furthermore we show that the principal functions corresponding to eigenvalues of $L(\lambda)$ belong to the space $L^{2}\left(\mathbf{R}_{+},\left\{\mathbf{C}_{2}\right)\right.$ and the principal functions corresponding to spectral singularities belong to a Hilbert space containing $L^{2}\left(\mathbf{R}_{+}, \mathbf{C}_{2}\right)$.
\end{abstract}

AMS Subject Classification : $34 L 05$ (34B05 47E05 34L40).

Key Words : Spectrum, Spectral Singularities, Non-Selfadjoint System of Differential Equations, 


\section{Introduction}

Let us consider the nonself-adjoint one dimensional Schrödinger operator $L$ generated in $L^{2}\left(\mathbf{R}_{+}\right)$by the differential expression

$$
\mathrm{l}(y)=-y^{\prime \prime}+q(x) y, \quad x \in \mathbf{R}_{+}
$$

and the boundary condition $y(0)=0$ as $L y=l y$, where $q$ is a complexvalued function. The spectral analysis of $L$ has been studied by Naimark [7]. Naimark has proved that there are some poles of resolvent's kernel which are not the eigenvalues of the operator L. (Schwartz [8] named these points as spectral singularities of $L$ ).Moreover Naimark has proved that spectral singularities are on the continuous spectrum, he has also shown that $L$ has a finite number of eigenvalues and spectral singularities with finite multiplicities if the condition

$$
\int_{0}^{\infty} e^{\varepsilon x}|q(x)| d x<\infty, \quad \varepsilon>0
$$

holds. Lyance has obtained the role of the spectral singularities in the spectral expansion of the operator $L$ in terms of principal functions[6].

The properties of the eigenvalues and vector-valued eigenfunctions of a boundary value problem for a one-dimensional Dirac system with a spectral parameter in the boundary conditions has been investigated by Kerimov [4].

We now consider the operator $L(\lambda)$ generated in $\mathrm{L}^{2}\left(\mathbf{R}_{+}, \mathbf{C}_{2}\right):=\left\{f(x): f(x)=\left(\begin{array}{l}f_{1}(x) \\ f_{2}(x)\end{array}\right), \int_{0}^{\infty}\left\{\left|f_{1}(x)\right|^{2}+\left|f_{2}(x)\right|^{2}\right\} d x<\infty\right\}$ by the system

$$
\begin{gathered}
i y_{1}^{\prime}+q_{1}(x) y_{2}=\lambda y_{1}, \\
-i y_{2}^{\prime}+q_{2}(x) y_{1}=\lambda y_{2}, \quad x \in \mathbf{R}_{+}
\end{gathered}
$$

and the spectral parameter-dependent boundary condition

$$
\left(a_{1} \lambda+b_{1}\right) y_{2}(0, \lambda)-\left(a_{2} \lambda+b_{2}\right) y_{1}(0, \lambda)=0
$$

where $q_{i}, i=1,2$, are complex-valued functions, $\lambda$ is the spectral parameter, $a_{i}, b_{i}$ are complex constants, $b_{i} \neq 0, i=1,2$; moreover $\left|a_{1}\right|^{2}+\left|a_{2}\right|^{2} \neq 0$. 
The spectrum of the operator generated by the system (1.1) with the boundary condition $y_{2}(0)-h y_{1}(0)=0$, (which is the special case of (1.2) when $\left.a_{i}=0, b=1\right)$ here $h \neq 0$ is a complex constant, has been investigated in [5] and in [1].

In this paper, we discussed the spectrum of $L(\lambda)$ defined by (1.1) and (1.2) and proved that $L(\lambda)$ has a finite number of eigenvalues and spectral singularities with finite multiplicities under the conditions

$$
\left|q_{i}(x)\right| \leq c e^{-\varepsilon x}<\infty, \quad i=1,2, \varepsilon>0, c>0
$$

by using analytic continuation method ([7]). Finally we observe the properties of the principal functions corresponding to eigenvalues and spectral singularities.

In the rest of the paper, we use the following notations:

$$
\begin{aligned}
& \mathbf{C}_{+}=\{\lambda: \lambda \in \mathbf{C}, \operatorname{Im} \lambda>0\}, \overline{\mathbf{C}}_{-}=\{\lambda: \lambda \in \mathbf{C}, \operatorname{Im} \lambda<0\}, \\
& \overline{\mathbf{C}}_{+}=\{\lambda: \lambda \in \mathbf{C}, \operatorname{Im} \lambda \geq 0\}, \overline{\mathbf{C}}_{-}=\{\lambda: \lambda \in \mathbf{C}, \operatorname{Im} \lambda \leq 0\},
\end{aligned}
$$

$\sigma_{p}(L(\lambda))$ denotes the eigenvalues and $\sigma_{s s}(L(\lambda))$ denotes the spectral singularities of $L(\lambda)$.

\section{Preliminaries}

Let us suppose that

$$
\left|q_{i}(x)\right| \leq c(1+x)^{-(1+\varepsilon)}, \quad i=1,2, \quad x \in \mathbf{R}_{+}, \quad \varepsilon>0
$$

holds, where $c>0$ is a constant.

The following results were given in [1] and in the first reference there in. Under the conditions (2.1), equation (1.1) has the following vector solutions

$$
e^{+}(x, \lambda)=\left(\begin{array}{c}
e_{1}^{+}(x, \lambda) \\
e_{2}^{+}(x, \lambda)
\end{array}\right)=\left(\begin{array}{c}
\int_{x}^{\infty} H_{12}(x, t) e^{i \lambda t} d t \\
e^{i \lambda x}+\int_{x}^{\infty} H_{22}(x, t) e^{i \lambda t} d t
\end{array}\right)
$$

for $\lambda \in \overline{\mathbf{C}}_{+}$and

$$
e^{-}(x, \lambda)=\left(\begin{array}{c}
e_{1}^{-}(x, \lambda) \\
e_{2}^{-}(x, \lambda)
\end{array}\right)=\left(\begin{array}{c}
e^{-i \lambda x}+\int_{x}^{\infty} H_{11}(x, t) e^{-i \lambda t} d t \\
\int_{x}^{\infty} H_{21}(x, t) e^{-i \lambda t} d t
\end{array}\right)
$$


for $\lambda \in \overline{\mathbf{C}}_{-}$; moreover the kernels $H_{i j}(x, t), i, j=1,2$, satisfy the inequalities

$$
\left|H_{i j}(x, t)\right| \leq c \sum_{k=1}^{2}\left|q_{k}\left(\frac{x+t}{2}\right)\right|,
$$

where $c>0$ is a constant. Therefore the functions $e_{i}^{+}(x, \lambda)$ and $e_{i}^{-}(x, \lambda)$, $i=1,2$, are analytic with respect to $\lambda$ in $\mathbf{C}_{+}, \mathbf{C}_{-}$, and continuous on $\overline{\mathbf{C}}_{+}$ and $\overline{\mathbf{C}}_{-}$, respectively. Moreover $e^{+}$and $e^{-}$satisfy the following asymptotic equalities ([1])

$$
e^{+}(x, \lambda)=\left(\begin{array}{c}
0 \\
e^{i \lambda x}
\end{array}\right)[1+o(1)], \quad \lambda \in \overline{\mathbf{C}}_{+} \quad x \rightarrow \infty
$$

and

$$
e^{-}(x, \lambda)=\left(\begin{array}{c}
e^{-i \lambda x} \\
0
\end{array}\right)[1+o(1)], \quad \lambda \in \overline{\mathbf{C}}_{-}, \quad x \rightarrow \infty .
$$

From (2.5) and (2.6) we have

$$
W\left\{e^{+}, e^{-}\right\}=\lim _{x \rightarrow \infty} W\left\{e^{+}(x, \lambda), e^{-}(x, \lambda)\right\}=-1
$$

for $\lambda \in \mathbf{R}$, where $W\left\{y^{(1)}, y^{(2)}\right\}$ is the wronskian of the solutions of $y^{(1)}$ and $y^{(2)}$ which is defined as $W\left\{y^{(1)}, y^{(2)}\right\}=y_{1}^{(1)} y_{2}^{(2)}-y_{1}^{(2)} y_{2}^{(1)}$, here $y^{(i)}=$ $\left(\begin{array}{l}y_{1}^{(i)} \\ y_{2}^{(i)}\end{array}\right), i=1,2$. Therefore $e^{+}, e^{-}$are the fundamental system of solutions of the system (1.1) for $\lambda \in \mathbf{R}$.

Let $\varphi(x, \lambda)$ be the solution of (1.1) satisfying the initial conditions

$$
\varphi_{1}(0, \lambda)=a_{1} \lambda+b_{1}, \quad \varphi_{2}(0, \lambda)=a_{2} \lambda+b_{2} .
$$

Clearly the solution $\varphi(x, \lambda)$ exists uniquely and is an entire function of $\lambda$..

\section{Eigenvalues and spectral singularities}

Let us define

$$
\begin{aligned}
& a^{+}(\lambda)=\left(a_{1} \lambda+b_{1}\right) e_{2}^{+}(0, \lambda)-\left(a_{2} \lambda+b_{2}\right) e_{1}^{+}(0, \lambda)=0 \\
& a^{-}(\lambda)=\left(a_{1} \lambda+b_{1}\right) e_{2}^{-}(0, \lambda)-\left(a_{2} \lambda+b_{2}\right) e_{1}^{-}(0, \lambda)=0 .
\end{aligned}
$$


Let

$$
R(x, t ; \lambda)=\left\{\begin{array}{l}
R^{+}(x, t ; \lambda), \operatorname{Im} \lambda \geq 0 \\
R^{-}(x, t ; \lambda), \operatorname{Im} \lambda \leq 0
\end{array}\right.
$$

be Green's function of $L(\lambda)$ which is obtained by using classical methods, here

$$
R^{+}(x, t ; \lambda)=\frac{i}{a^{+}(\lambda)}\left\{\begin{array}{c}
e^{+}(x, \lambda) \varphi^{*}(t, \lambda), 0 \leq t \leq x \\
\varphi(x, \lambda)\left(e^{+}\right)^{*}(t, \lambda), x<t \leq \infty
\end{array}\right.
$$

and

$$
R^{-}(x, t ; \lambda)=\frac{i}{a^{-}(\lambda)}\left\{\begin{array}{c}
e^{-}(x, \lambda) \varphi^{*}(t, \lambda), 0 \leq t \leq x \\
\varphi(x, \lambda)\left(e^{-}\right)^{*}(t, \lambda), x<t \leq \infty
\end{array}\right.
$$

and $\left(e^{ \pm}\right)^{*}:=\left(e_{2}^{ \pm}, e_{1}^{ \pm}\right), \varphi^{*}:=\left(\varphi_{2}, \varphi_{1}\right)$. Moreover from (2.5) and (2.6) we have

$$
e^{+}(x, \lambda) \in L^{2}\left(\mathbf{R}_{+}, \mathbf{C}_{2}\right)
$$

for $\lambda \in \mathbf{C}_{+}$and

$$
e^{-}(x, \lambda) \in L^{2}\left(\mathbf{R}_{+}, \mathbf{C}_{2}\right)
$$

for $\lambda \in \mathbf{C}_{-}$.In this case we state the following

\section{Lemma 3.1.}

a) $\sigma_{p}(L(\lambda))=\left\{\lambda: \lambda \in \mathbf{C}_{+}, a^{+}(\lambda)=0\right\} \cup\left\{\lambda: \lambda \in \mathbf{C}_{-}, a^{-}(\lambda)=0\right\}$,

b) $\sigma_{s s}(L(\lambda))$

$=\left\{\lambda: \lambda \in \mathbf{R} \backslash\{0\}, a^{+}(\lambda)=0\right\} \cup\left\{\lambda: \lambda \in \mathbf{R} \backslash\{0\}, a^{-}(\lambda)=0\right\}$.

Proof. a) It is clear that

$$
\left\{\lambda: \lambda \in \mathbf{C}_{+}, a^{+}(\lambda)=0\right\} \cup\left\{\lambda: \lambda \in \mathbf{C}_{-}, a^{-}(\lambda)=0\right\} \subset \sigma_{p}(L(\lambda)) .
$$

Now let us suppose that $\lambda_{0} \in \sigma_{p}(L(\lambda))$. If $\lambda_{0} \in \mathbf{C}_{+}$then (1.1) has a nontrivial solution $y\left(x, \lambda_{0}\right)$ in $L^{2}\left(\mathbf{R}_{+}, \mathbf{C}_{2}\right)$ for $\lambda=\lambda_{0}$ satisfying (1.2).

Since $W\left\{y\left(x, \lambda_{0}\right), \varphi\left(x, \lambda_{0}\right)\right\}=0$ then there exists a constant $c \neq 0$ such that $y\left(x, \lambda_{0}\right)=c \varphi\left(x, \lambda_{0}\right)$. Therefore

$$
\begin{aligned}
& W\left\{y\left(x, \lambda_{0}\right), e^{+}\left(x, \lambda_{0}\right)\right\} \\
& =y_{1}\left(0, \lambda_{0}\right) e_{2}^{+}\left(0, \lambda_{0}\right)-y_{2}\left(0, \lambda_{0}\right) e_{1}^{+}\left(0, \lambda_{0}\right)=c a^{+}\left(\lambda_{0}\right) .
\end{aligned}
$$

Moreover we find from (3.5) that 


$$
\begin{array}{r}
W\left\{y\left(x, \lambda_{0}\right), e^{+}\left(x, \lambda_{0}\right)\right\} \\
=\lim _{x \rightarrow \infty}\left\{y_{1}\left(x, \lambda_{0}\right) e_{2}^{+}\left(x, \lambda_{0}\right)-y_{2}\left(x, \lambda_{0}\right) e_{1}^{+}\left(x, \lambda_{0}\right)\right\} \\
=0
\end{array}
$$

So we obtain from (3.7) and (3.8) that $a^{+}\left(\lambda_{0}\right)=0$.

If $\lambda_{0} \in \mathbf{C}_{-}$then we prove that $a^{-}\left(\lambda_{0}\right)=0$ similarly.

If $\lambda_{0} \in \mathbf{R}$, then the general solution of (1.1) is

$\mathrm{y}\left(x, \lambda_{0}\right)=c_{1} e^{+}\left(x, \lambda_{0}\right)+c_{2} e^{-}\left(x, \lambda_{0}\right)$

for $\lambda=\lambda_{0}$. From (2.5) and (2.6) we have

$$
\mathrm{y}\left(x, \lambda_{0}\right)=\left(\begin{array}{c}
c_{2} e^{-i \lambda_{0} x} \\
c_{1} e^{i \lambda_{0} x}
\end{array}\right)(1+o(1))
$$

as $x \rightarrow \infty$. Therefore $y\left(x, \lambda_{0}\right) \notin L^{2}\left(\mathbf{R}_{+}, \mathbf{C}_{2}\right)$. Hence $\sigma_{p}(L(\lambda)) \cap \mathbf{R}=$, so (a) follows.

b) Spectral singularities which are not the eigenvalues of $L(\lambda)$, are the poles of the resolvent's kernel. From (3.1) - (3.4) and $(a)$, we can say that the spectral singularities of $L(\lambda)$ are the real zeros of $a^{+}$and $a^{-}$. So (b) follows.

Furthermore

$$
\mathrm{W}\left\{e^{+}(x, \lambda), e^{-}(x, \lambda)\right\}=e_{1}^{+}(0, \lambda) e_{2}^{-}(0, \lambda)-e_{2}^{+}(0, \lambda) e_{1}^{-}(0, \lambda)=-1
$$

for $\lambda \in \mathbf{R}$. Therefore we have

$$
\left\{\lambda: \lambda \in \mathbf{R}, a^{+}(\lambda)=0\right\} \cap\left\{\lambda: \lambda \in \mathbf{R}, a^{-}(\lambda)=0\right\}=\phi
$$

Now as we see from Lemma 3.1 that to investigate the properties of the eigenvalues and the spectral singularities of $L(\lambda)$, we need to investigate the properties of the zeros of $a^{+}$and $a^{-}$in $\overline{\mathbf{C}}_{+}, \overline{\mathbf{C}}_{-}$, respectively. For simplicity, we will consider only the zeros of $a^{+}$in $\overline{\mathbf{C}}_{+}$. In this point of view let us define the sets $Z_{+}=\left\{\lambda: \lambda \in \mathbf{C}_{+}, a^{+}(\lambda)=0\right\}$, $Z=\left\{\lambda: \lambda \in \mathbf{R}, a^{+}(\lambda)=0\right\}$.

Lemma 3.2. (a) The set $Z_{+}$is bounded and has at most a countable number of elements, and its limit points can lie only in a bounded subinterval of the real axis.

(b) $Z$ is a compact set. 
Proof. From (2.2) we get that $a^{+}(\lambda)$ is analytic in $\mathbf{C}_{+}$and satisfies

$$
\begin{aligned}
& a^{+}(\lambda)=a_{1} \lambda+b_{1}+ \\
& \int_{0}^{\infty}\left\{\left(a_{1} \lambda+b_{1}\right) H_{22}(0, t)-\left(a_{2} \lambda+b_{2}\right) H_{12}(0, t)\right\} e^{i \lambda t} d t .
\end{aligned}
$$

From (3.10) we get

$$
\begin{aligned}
& a^{+}(\lambda)=\lambda\left(a_{1}+\int_{0}^{\infty}\left\{a_{1} H_{22}(0, t)-a_{2} H_{12}(0, t)\right\} e^{i \lambda t} d t\right) \\
& +O(1)
\end{aligned}
$$

for $\lambda \in \overline{\mathbf{C}}_{+},|\lambda| \rightarrow \infty$. From (3.11) we find that the zeros of $a^{+}$must lie in a bounded domain. Since $a^{+}$is analytic in $\mathbf{C}_{+}$then these zeros are at most countable numbers. From the uniqueness of analytic functions the limit points of $Z_{+}$can lie only in a bounded subinterval of the real axis. So $(a)$ follows. (b) is obtained from the uniqueness theorem of analytic functions [3]

From Lemma 3.1 and Lemma 3.2 we have

Theorem 3.3. If the conditions (2.1) hold, then the set of eigenvalues and spectral singularities of $L(\lambda)$ are bounded, countable and their limit points can lie only in a bounded subinterval of the real axis.

Definition 3.4. The multiplicity of a zero of $a^{+}$(or $a^{-}$) in $\overline{\mathbf{C}}_{+}$(or $\left.\overline{\mathbf{C}}_{-}\right)$ is defined as the multiplicity of the corresponding eigenvalue or spectral singularity of $L(\lambda)$.

Let us suppose that

$$
\left|q_{i}(x)\right| \leq c e^{-\varepsilon x}, \quad c>0, \quad \varepsilon>0, \quad i=1,2
$$

hold. From (2.4) we obtain that

$$
\left|H_{i j}(x, t)\right| \leq c \exp \left\{\frac{-\varepsilon}{2}(x+t)\right\} .
$$

From (3.10) and (3.13),$a^{+}$has an analytic continuation from the real axis to the half plane $\operatorname{Im} \lambda>-\frac{\varepsilon}{2}$. So the limit points of the sets $Z_{+}$and $Z$ cannot lie in $\mathbf{R}$ i.e. the sets $Z_{+}$and $Z$ have no limit points. Therefore the number of zeros of $a^{+}$in $\overline{\mathbf{C}}_{+}$are finite with finite multiplicities. Similarly we can 
show that $a^{-}$has a finite number of zeros with finite multiplicities in $\overline{\mathbf{C}}_{-}$.So we have proved the following

Theorem 3.5. The operator $L(\lambda)$ has a finite number of eigenvalues and spectral singularities, and each of them is of finite multiplicity if the conditions (3.12) hold.

\section{Principal functions}

Assume that (3.12) holds. Let $\lambda_{1}^{+}, \ldots, \lambda_{j}^{+}$and $\lambda_{1}^{-}, \ldots, \lambda_{k}^{-}$denote the zeros of $a^{+}$in $\mathbf{C}_{+}$and $a^{-}$in $\mathbf{C}_{-}$with multiplicities $m_{1}^{+}, \ldots, m_{j}^{+}$and $m_{1}^{-}, \ldots, m_{k}^{-}$, respectively. Similarly, let $\lambda_{1}, \ldots, \lambda_{p}$ and $\lambda_{p+1}, \ldots, \lambda_{q}$ denote the zeros of $a^{+}$and $a^{-}$on the real axis with multiplicities $m_{1}, \ldots, m_{p}$ and $m_{p+1}, \ldots, m_{q}$, respectively. In this case we have

$$
\left\{\frac{\partial^{n}}{\partial \lambda^{n}} W\left[\varphi(x, \lambda), e^{+}(x, \lambda)\right]\right\}_{\lambda=\lambda_{i}^{+}}=\left\{\frac{d^{n}}{d \lambda^{n}} a^{+}(\lambda)\right\}_{\lambda=\lambda_{i}^{+}}=0
$$

for $n=0,1, \ldots, m_{i}^{+}-1, i=1,2, \ldots, j$, and

$$
\left\{\frac{\partial^{n}}{\partial \lambda^{n}} W\left[\varphi(x, \lambda), e^{-}(x, \lambda)\right]\right\}_{\lambda=\lambda_{l}^{-}}=\left\{\frac{d^{n}}{d \lambda^{n}} a^{-}(\lambda)\right\}_{\lambda=\lambda_{l}^{-}}=0
$$

for $n=0,1, \ldots, m_{l}^{-}-1, l=1,2, \ldots, k$. Clearly we have

$$
\begin{aligned}
& \varphi\left(x, \lambda_{i}^{+}\right)=c_{0}\left(\lambda_{i}^{+}\right) e^{+}\left(x, \lambda_{i}^{+}\right), i=1,2, \ldots, j, \\
& \varphi\left(x, \lambda_{l}^{-}\right)=d_{0}\left(\lambda_{l}^{-}\right) e^{-}\left(x, \lambda_{l}^{-}\right), l=1,2, \ldots, k,
\end{aligned}
$$

when $n=0$. Therefore $c_{0}\left(\lambda_{i}^{+}\right) \neq 0, d_{0}\left(\lambda_{l}^{-}\right) \neq 0$. Therefore we can state the following lemma

Theorem 4.1. The following equalities

$$
\left\{\frac{\partial^{n}}{\partial \lambda^{n}} \varphi(x, \lambda)\right\}_{\lambda=\lambda_{i}^{+}}=\sum_{v=0}^{n}\left(\begin{array}{l}
n \\
v
\end{array}\right) c_{n-v}^{+}\left(\lambda_{i}^{+}\right)\left\{\frac{\partial^{v}}{\partial \lambda^{v}} e^{+}(x, \lambda)\right\}_{\lambda=\lambda_{i}^{+}}
$$

for $n=0,1, \ldots, m_{i}^{+}-1, i=1,2, \ldots, j$ and

$$
\left\{\frac{\partial^{n}}{\partial \lambda^{n}} \varphi(x, \lambda)\right\}_{\lambda=\lambda_{l}^{-}}=\sum_{v=0}^{n}\left(\begin{array}{l}
n \\
v
\end{array}\right) d_{n-v}^{-}\left(\lambda_{l}^{-}\right)\left\{\frac{\partial^{v}}{\partial \lambda^{v}} e^{-}(x, \lambda)\right\}_{\lambda=\lambda_{l}^{-}}
$$


for $n=0,1, \ldots, m_{l}^{-}-1, l=1,2, \ldots, k$ hold, where the constants $c_{0}^{+}, c_{1}^{+}, \ldots, c_{n}^{+}$ and $d_{0}^{-}, d_{1}^{-}, \ldots, d_{n}^{-}$depend on $\lambda_{i}^{+}$and $\lambda_{l}^{-}$, respectively.

Proof. Using mathematical induction, we prove first (4.5). For $n=$ 0 , The proof is clear by (4.3). Now we suppose that (4.5) holds for $1 \leq$ $n_{0} \leq m_{i}^{+}-2$, i.e.

$$
\text { (4.7) }\left\{\frac{\partial^{n_{0}}}{\partial \lambda^{n_{0}}} \varphi(x, \lambda)\right\}_{\lambda=\lambda_{i}^{+}}=\sum_{v=0}^{n_{0}}\left(\begin{array}{c}
n_{0} \\
v
\end{array}\right) c_{n_{0}-v}^{+}\left(\lambda_{i}^{+}\right)\left\{\frac{\partial^{v}}{\partial \lambda^{v}} e^{+}(x, \lambda)\right\}_{\lambda=\lambda_{i}^{+}} .
$$

Now we will show that (4.5) also holds for $n_{0}+1$. If $U(x, \lambda)$ is a solution of the equation (1.1), then $\frac{\partial^{n}}{\partial \lambda^{n}} U(x, \lambda)$ satisfies the following equation:

$$
\left\{J \frac{d}{d x}+Q(x)-\lambda\right\} \frac{\partial^{n}}{\partial \lambda^{n}} U(x, \lambda)=n \frac{\partial^{n-1}}{\partial \lambda^{n-1}} U(x, \lambda),
$$

where

$$
\mathrm{J}=\left[\begin{array}{cc}
i & 0 \\
0 & -i
\end{array}\right], \quad Q(x)=\left[\begin{array}{rr}
0 & q_{1}(x) \\
q_{2}(x) & 0
\end{array}\right], \quad U(x, \lambda)=\left[\begin{array}{l}
U_{1}(x, \lambda) \\
U_{2}(x, \lambda)
\end{array}\right] .
$$

Writing (4.8) for $\varphi\left(x, \lambda_{i}^{+}\right)$and $e^{+}\left(x, \lambda_{i}^{+}\right)$, then using (4.7), we get

$$
\left\{J \frac{d}{d x}+Q(x)-\lambda_{i}^{+}\right\} f_{n_{0}+1}\left(x, \lambda_{i}^{+}\right)=0,
$$

where

$$
\begin{aligned}
f_{n_{0}+1}\left(x, \lambda_{i}^{+}\right)= & \left\{\frac{\partial^{n_{0}+1}}{\partial \lambda^{n_{0}+1}} \varphi(x, \lambda)\right\}_{\lambda=\lambda_{i}^{+}} \\
& -\sum_{v=1}^{n_{0}+1}\left(\begin{array}{c}
n_{0}+1 \\
v
\end{array}\right) c_{n_{0}+1-v}^{+}\left(\lambda_{i}^{+}\right)\left\{\frac{\partial^{v}}{\partial \lambda^{v}} e^{+}(x, \lambda)\right\}_{\lambda=\lambda_{i}^{+}} .
\end{aligned}
$$

therefore we have

$$
W\left[f_{n_{0}+1}\left(x, \lambda_{i}^{+}\right), e^{+}\left(x, \lambda_{i}^{+}\right)\right]=\left\{\frac{\partial^{n_{0}+1}}{\partial \lambda^{n_{0}+1}} W\left[\varphi(x, \lambda), e^{+}(x, \lambda)\right\}_{\lambda=\lambda_{i}^{+}}=0\right.
$$

by (4.1). Hence there exists a constant $c_{n_{0}+1}^{+}\left(\lambda_{i}^{+}\right)$such that 


$$
\mathrm{f}_{n_{0}+1}\left(x, \lambda_{i}^{+}\right)=c_{n_{0}+1}^{+}\left(\lambda_{i}^{+}\right) e^{+}\left(x, \lambda_{i}^{+}\right)
$$

which proves the theorem. Similarly we can prove that (4.6) holds.

Now we introduce the principal functions corresponding to the eigenvalues as follows:

$$
\begin{aligned}
& \left\{\frac{\partial^{n}}{\partial \lambda^{n}} \varphi(x, \lambda)\right\}_{\lambda=\lambda_{i}^{+}}, \quad n=0,1, \ldots, m_{i}^{+}-1, i=1,2, \ldots, j, \\
& \left\{\frac{\partial^{n}}{\partial \lambda^{n}} \varphi(x, \lambda)\right\}_{\lambda=\lambda_{l}^{-}}, \quad n=0,1, \ldots, m_{l}^{-}-1, l=1,2, \ldots, k
\end{aligned}
$$

are called the principal functions corresponding to the eigenvalues $\lambda=$ $\lambda_{i}^{+}, i=1,2, \ldots, j$ and $\lambda=\lambda_{l}^{-}, l=1,2, \ldots, k$ of $L(\lambda)$, respectively.

Therefore we arrive at the following result for the principal functions given above:

Theorem 4.2. The principal functions corresponding to the eigenvalues of $L(\lambda)$ are in $L^{2}\left(\mathbf{R}_{+}, \mathbf{C}_{2}\right)$, i.e.:

$$
\begin{aligned}
& \left\{\frac{\partial^{n}}{\partial \lambda^{n}} \varphi(., \lambda)\right\}_{\lambda=\lambda_{i}^{+}} \in L^{2}\left(\mathbf{R}_{+}, \mathbf{C}_{2}\right), \\
& n=0,1, \ldots, m_{i}^{+}-1, i=1,2, \ldots, j, \\
& \left\{\frac{\partial^{n}}{\partial \lambda^{n}} \varphi(., \lambda)\right\}_{\lambda=\lambda_{l}^{-}} \in L^{2}\left(\mathbf{R}_{+}, \mathbf{C}_{2}\right), \\
& n=0,1, \ldots, m_{l}^{-}-1, l=1,2, \ldots, k .
\end{aligned}
$$

Proof. From (3.13) and (2.2) we obtain that

$$
\mid \begin{aligned}
& \left\{\frac{\partial^{n}}{\partial \lambda^{n}} e_{1}^{+}(x, \lambda)\right\}_{\lambda=\lambda_{i}^{+}} \mid \leq c_{1} e^{-\varepsilon x}, \\
& \left\{\frac{\partial^{n}}{\partial \lambda^{n}} e_{2}^{+}(x, \lambda)\right\}_{\lambda=\lambda_{i}^{+}} \mid \leq x^{n} e^{-x \operatorname{Im} \lambda_{i}^{+}+c_{2} e^{-\varepsilon x}}
\end{aligned}
$$

for $n=0,1, \ldots, m_{i}^{+}-1, i=1,2, \ldots, j$ which gives (4.9) by using (4.5). Equation (4.10) may be derived, by using (4.6), analogously.

\section{Definition 4.3.}

Obviously we also have

$$
\left\{\frac{\partial^{n}}{\partial \lambda^{n}} W\left[\varphi(x, \lambda), e^{+}(x, \lambda)\right\}_{\lambda=\lambda_{i}}=\left\{\frac{d^{n}}{d \lambda^{n}} a^{+}(\lambda)\right\}_{\lambda=\lambda_{i}}=0\right.
$$

for $n=0,1, \ldots, m_{i}-1, i=1,2, \ldots, p$ and 


$$
\left\{\frac{\partial^{n}}{\partial \lambda^{n}} W\left[\varphi(x, \lambda), e^{-}(x, \lambda)\right\}_{\lambda=\lambda_{l}}=\left\{\frac{d^{n}}{d \lambda^{n}} a^{-}(\lambda)\right\}_{\lambda=\lambda_{l}}=0\right.
$$

for $n=0,1, \ldots, m_{l}-1, l=p+1, p+2, \ldots, q$. Using the last two formulas given above, in a similar way to Theorem 4.1 we get that

Remark 4.3. The formulas

$$
\left\{\frac{\partial^{n}}{\partial \lambda^{n}} \varphi(x, \lambda)\right\}_{\lambda=\lambda_{i}}=\sum_{v=0}^{n}\left(\begin{array}{l}
n \\
v
\end{array}\right) c_{n-v}\left(\lambda_{i}\right)\left\{\frac{\partial^{v}}{\partial \lambda^{v}} e^{+}(x, \lambda)\right\}_{\lambda=\lambda_{i}}
$$

for $n=0,1, \ldots, m_{i}-1, i=1,2, \ldots, p$, and

$$
\left\{\frac{\partial^{n}}{\partial \lambda^{n}} \varphi(x, \lambda)\right\}_{\lambda=\lambda_{l}}=\sum_{v=0}^{n}\left(\begin{array}{l}
n \\
v
\end{array}\right) d_{n-v}\left(\lambda_{l}\right)\left\{\frac{\partial^{v}}{\partial \lambda^{v}} e^{-}(x, \lambda)\right\}_{\lambda=\lambda_{l}}
$$

for $n=0,1, \ldots, m_{l}-1, l=p+1, p+2, \ldots, q$ hold, where the constants $c_{0}, c_{1}, \ldots, c_{n}$ and $d_{0}, d_{1}, \ldots, d_{n}$ depend on $\lambda_{i}$ and $\lambda_{l}$, respectively.

Now we introduce the principal functions corresponding to the spectral singularities as follows:

$$
\begin{aligned}
& \left\{\frac{\partial^{n}}{\partial \lambda^{n}} \varphi(x, \lambda)\right\}_{\lambda=\lambda_{i}}, \quad n=0,1, \ldots, m_{i}-1, i=1,2, \ldots, p, \\
& \left\{\frac{\partial^{n}}{\partial \lambda^{n}} \varphi(x, \lambda)\right\}_{\lambda=\lambda_{l}}, \quad n=0,1, \ldots, m_{l}-1, l=p+1, p+2, \ldots, q
\end{aligned}
$$

are called the principal functions corresponding to the spectral singularities $\lambda=\lambda_{i} i=1,2, \ldots, p$ and $\lambda=\lambda_{l} l=p+1, p+2, \ldots, q$ of $L(\lambda)$, respectively. Therefore we arrive at the following

Lemma 4.4 The principal functions for the spectral singularities do not belong to the space $L^{2}\left(\mathbf{R}_{+}, \mathbf{C}_{2}\right)$, i.e: $\left\{\frac{\partial^{n}}{\partial \lambda^{n}} \varphi(., \lambda)\right\}_{\lambda=\lambda_{i}} \notin L^{2}\left(\mathbf{R}_{+}, \mathbf{C}_{2}\right)$, for $n=0,1, \ldots, m_{i}-1, i=1,2, \ldots, p,\left\{\frac{\partial^{n}}{\partial \lambda^{n}} \varphi(., \lambda)\right\}_{\lambda=\lambda_{l}} \notin L^{2}\left(\mathbf{R}_{+}, \mathbf{C}_{2}\right)$, for $n=0,1, \ldots, m_{l}-1, l=p+1, p+2, \ldots, q$.

The proof of the lemma is obtained from (2.2), (2.3), (4.11) and (4.12).

Now let us introduce the following Hilbert spaces [2]

$$
\begin{aligned}
H\left(\mathbf{R}_{+}, \mathbf{C}_{2}, m\right):= & \left\{f(x): f(x)=\left(\begin{array}{l}
f_{1}(x) \\
f_{2}(x)
\end{array}\right),\right. \\
& \left.\int_{0}^{\infty}(1+x)^{2 m}\left\{\left|f_{1}(x)\right|^{2}+\left|f_{2}(x)\right|^{2}\right\} d x<\infty\right\},
\end{aligned}
$$


$m=0,1, \ldots$ with norm

$$
\|f\|_{H\left(\mathbf{R}_{+}, \mathbf{C}_{2}, m\right)}^{2}=\int_{0}^{\infty}(1+x)^{2 m}\left\{\left|f_{1}(x)\right|^{2}+\left|f_{2}(x)\right|^{2}\right\} d x
$$

and

$$
\begin{aligned}
H\left(\mathbf{R}_{+}, \mathbf{C}_{2},-m\right): & =\left\{g(x): g(x)=\left(\begin{array}{l}
g_{1}(x) \\
g_{2}(x)
\end{array}\right),\right. \\
& \left.\int_{0}^{\infty}(1+x)^{-2 m}\left\{\left|g_{1}(x)\right|^{2}+\left|g_{2}(x)\right|^{2}\right\} d x<\infty\right\},
\end{aligned}
$$

$m=0,1, \ldots$ with norm

$$
\|g\|_{H\left(\mathbf{R}_{+}, \mathbf{C}_{2},-m\right)}^{2}=\int_{0}^{\infty}(1+x)^{-2 m}\left\{\left|g_{1}(x)\right|^{2}+\left|g_{2}(x)\right|^{2}\right\} d x .
$$

Clearly $H\left(\mathbf{R}_{+}, \mathbf{C}_{2}, 0\right)=L^{2}\left(\mathbf{R}_{+}, \mathbf{C}_{2}\right)$ and

$$
\mathrm{H}\left(\mathbf{R}_{+}, \mathbf{C}_{2}, m\right) L^{2}\left(\mathbf{R}_{+}, \mathbf{C}_{2},\right) H\left(\mathbf{R}_{+}, \mathbf{C}_{2},-m\right) .
$$

Therefore we reach to the following

\section{Theorem 4.5.}

$$
\left\{\frac{\partial^{n}}{\partial \lambda^{n}} \varphi(., \lambda)\right\}_{\lambda=\lambda_{i}} \in H\left(\mathbf{R}_{+}, \mathbf{C}_{2},-(n+1)\right),
$$

for $n=0,1, \ldots, m_{i}-1, i=1,2, \ldots, p$, and

$$
\left\{\frac{\partial^{n}}{\partial \lambda^{n}} \varphi(., \lambda)\right\}_{\lambda=\lambda_{l}} \in H\left(\mathbf{R}_{+}, \mathbf{C}_{2},-(n+1)\right)
$$

for $n=0,1, \ldots, m_{l}-1, l=p+1, p+2, \ldots, q$.

Proof. From (2.2), we obtain that

$$
\left|\left\{\frac{\partial^{n}}{\partial \lambda^{n}} e_{1}^{+}(x, \lambda)\right\}_{\lambda=\lambda_{i}}\right| \leq \int_{x}^{\infty} t^{n}\left|H_{12}(x, t)\right| d t
$$


and

$$
\left|\left\{\frac{\partial^{n}}{\partial \lambda^{n}} e_{2}^{+}(x, \lambda)\right\}_{\lambda=\lambda_{i}}\right| \leq x^{n}+\int_{x}^{\infty} t^{n}\left|H_{22}(x, t)\right| d t .
$$

for $n=0,1, \ldots, m_{i}-1, i=1,2, \ldots, p$. By the definition of $H\left(\mathbf{R}_{+}, \mathbf{C}_{2},-(n+1)\right)$ and using (4.15) and (4.16) we arrive at (4.13). In a similar way, we can show that (4.14) also holds.

Now let us choose $n_{0}$ so that

$$
\mathrm{n}_{0}=\max \left\{m_{1}, \ldots, m_{p}, m_{p+1}, \ldots, m_{q}\right\} .
$$

Then

$$
H\left(\mathbf{R}_{+}, \mathbf{C}_{2}, n_{0}\right) \underset{\neq}{\subset} L^{2}\left(\mathbf{R}_{+}, \mathbf{C}_{2},\right) \underset{\neq}{\subsetneq} H\left(\mathbf{R}_{+}, \mathbf{C}_{2},-n_{0}\right) .
$$

From Theorem 4.5, we finally reach to the following

Conclusion 4.6. The principal functions for the spectral singularities of the operator $L(\lambda)$ belong to the space $H\left(\mathbf{R}_{+}, \mathbf{C}_{2},-n_{0}\right)$, i.e.:

$$
\left\{\frac{\partial^{n}}{\partial \lambda^{n}} \varphi(., \lambda)\right\}_{\lambda=\lambda_{i}} \in H\left(\mathbf{R}_{+}, \mathbf{C}_{2},-n_{0}\right)
$$

for $n=0,1, \ldots, m_{i}-1, i=1,2, \ldots, p$ and

$$
\left\{\frac{\partial^{n}}{\partial \lambda^{n}} \varphi(., \lambda)\right\}_{\lambda=\lambda_{l}} \in H\left(\mathbf{R}_{+}, \mathbf{C}_{2},-n_{0}\right)
$$

for $n=0,1, \ldots, m_{l}-1, l=p+1, p+2, \ldots, q$.

\section{References}

[1] Ö. Akın and E. Bairamov, On the structure of discrete spectrum of the non-selfadjoint system of differential equations in the first order, J. Korean Math. Soc. No 3, 32, pp. 401-413, (1995).

[2] Yu. M. Berezanski, Expansion in Eigenfunctions of Selfadjoint Operators, Amer. Math. Soc., Providence R. I. (1968). 
[3] E. P. Dolzhenko, Boundary Value Uniqueness Theorems for Analytic Functions, Math. Notes 25 No 6, pp. 437-442, (1979).

[4] N. B. Kerimov, A Boundary Value Problem for the Dirac System with a Spectral Parameter in the Boundary Conditions, Differential Equations, Vol. 38, No 2, pp. 164-174, (2002).

[5] E. Kır, Spectral Properties of Non-Selfadjoint System of Differential Equations, Commun. Fac. Sci. Univ. Ank. Series A1, Vol.49 (2000),111116.

[6] V.E. Lyance, A differential Operator with Spectral Singularities, I,II, Amer. Math. Soc.Trans. Ser. 2, Vol. 60, pp. 185-225, pp. 227-283, (1967).

[7] M.A.Naimark, Investigation of the Spectrum and the Expansion in Eigenfunctions of a Non-selfadjoint Operator of Second Order on a Semi-axis, Amer. Math. Soc. Trans. Ser. 2, Vol 16, pp.103-193, Amer. Math. Soc., Providence, (1960).

[8] J.T.Schwartz, Some non-selfadjoint operators, Comm. Pure and Appl.Math. 13, pp. 609-639, (1960).

\author{
Esra Kir \\ Faculty of Art and Science \\ 06500, Teknikokullar \\ Ankara \\ Turkey \\ e-mail : esrakir@gazi.edu.tr
}




\author{
Gülen Başcanbaz-Tunca \\ Department of mathematics \\ Faculty of science \\ Ankara University \\ 06100, Tandogan, ankara \\ Turkey \\ e-mail: tunca@science.ankara.edu.tr \\ and
}

\title{
Canan Yanik
}

Department of Mathematics Education

Hacettepe University

06532 Beytepe

Ankara

Turkey

e-mail : canany@hacettepe.edu.tr 